\title{
TEMA 12-2014: Síndrome Compartimental Abdominal
}
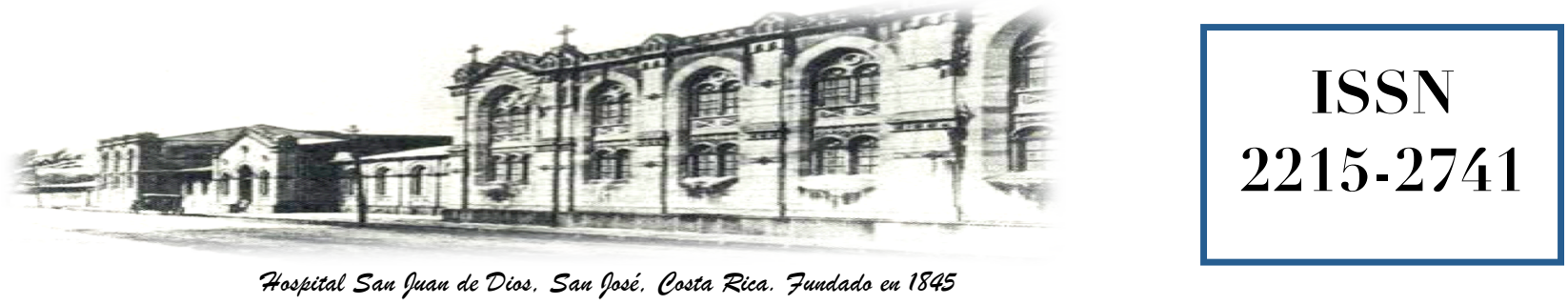

Recibido:

$06 / 02 / 2014$

Aceptado:

$24 / 04 / 2014$

Ronny Granados Quesada ${ }^{1}$ Julia Vargas Carranza ${ }^{2}$

${ }^{1}$ Médico Cirujano Universidad de Ciencias Médicas. Servicio de Cirugía Ambulatoria. Hospital San Juan de Dios. Correo electrónico: ronnygq10@hotmail.com

${ }^{2}$ Medica Asistente Especialista en Cirugía General. Jefe Servicio Cirugía 1 y Cirugía Ambulatoria. Hospital San Juan de Dios.

\section{RESUMEN}

El seguimiento adecuado de los pacientes críticos médico-quirúrgicos facilita el diagnóstico temprano y el tratamiento adecuado de la hipertensión intraabdominal (HIA) y del síndrome compartimental abdominal (SCA). La cavidad abdominal y el retroperitoneo actúan como compartimentos cerrados y cualquier cambio en el volumen de su contenido puede elevar la presión intraabdominal (PIA). La HIA es sólo una medida elevada de la PIA y el SCA supone el punto final de una HIA sostenida, con la aparición de disfunción orgánica. Para el diagnóstico de la HIA y del SCA se requiere medir la PIA, la presión de perfusión abdominal y el $\mathrm{pH}$ intramucoso gástrico, para correlacionar estos datos con signos de deterioro clínico en el paciente. Las medidas terapéuticas médicas en el SCA son limitadas y la descompresión abdominal es el tratamiento del SCA sintomático establecido.

\section{PALABRAS CLAVE}

Presión intraabdominal. intraabdominal. Síndrome abdominal.
Hipertensión compartimental

\begin{abstract}
Correct monitoring of medico-surgical criticallyill patients aids the early diagnosis and appropriate treatment of intra-abdominal hypertension (IAH) and abdominal compartment syndrome (ACS). The abdominal cavity and the retroperitoneum act closed compartments and any change in the volume of their contents can increase intraabdominal pressure (IAP). IAH is only one measure of elevated IAP, and ACS represents the end result of sustained IAH with the appearance of organ dysfunction. To diagnose IAH and ACS, measurement of IAP, abdominal perfusion pressure and intramucous gastric $\mathrm{pH}$ must be performed and the results correlated with signs of clinical deteriorationin the patient. Medical therapeutic measures in ACS are limited and abdominal decompression is the established symptomatic treatment of this entity.
\end{abstract}

\section{KEY WORDS}

Intra-abdominal pressure. Intra-abdominal

hypertension. Abdominal compartment syndrome. 


\section{INTRODUCCIÓN}

Durante la segunda mitad del siglo XIX surge el interés por medir la presión intraabdominal (PIA), para correlacionar su aumento con las alteraciones fisiológicas que ponen en peligro la función de algunos órganos en pacientes críticos médico-quirúrgicos. En 1984 se comunica la primera serie clínica con medida de la PIA transvesical, se establecen las indicaciones para la descompresión abdominal y se introduce el concepto de síndrome compartimental abdominal (SCA).

El mejor conocimiento de la fisiopatología y el seguimiento adecuado de los pacientes ingresados en las unidades de cuidados intensivos (UCI) facilitan el diagnóstico temprano de la hipertensión intraabdominal (HIA) y del SCA, lo que permite un tratamiento adecuado para prevenir el establecimiento y la progresión de un síndrome de disfunción multiorgánica $(\mathrm{SDMO})^{(1)}$.

La conferencia internacional de consenso sobre HIA y SCA, celebrada en Noosa (Queensland, Australia) en 2004, marca un antes y un después, ya que se unifican los criterios de concepto, diagnóstico y tratamiento de estos pacientes, lo que permite hablar el mismo idioma y poder contrastar los resultados ${ }^{(2)}$.

La introducción de las técnicas quirúrgicas por laparoscopia, al provocar un aumento de presión intraabdominal y simular así un estado de HIA patológico, supuso un claro en el mar de oscuridad que rodeaba a esta entidad, siendo Kron y sus colaboradores quienes publicaron en 1984 las primeras series de mediciones de la PIA y quienes acunaron por primera vez el término de SCA, considerándolo como criterio de tratamiento quirúrgico ${ }^{(3)}$.

La PIA es un parámetro de gran importancia en el paciente crítico, siendo un indicador pronóstico de su estado fisiopatológico, por lo que se ha de convertir en una monitorización rutinaria en determinados pacientes.

La PIA normal, en reposo, se suele mover en unos valores que van desde los subatmosféricos hasta valores próximos a $5-6.5 \mathrm{mmHg}$. Tiene una gran variabilidad, dependiendo de la posición, del hábito corporal y de la actividad, fluctuando con el ciclo respiratorio (aumentando con la inspiración por la acción del diafragma y disminuyendo con la espiración) y pudiendo alcanzar incluso los $300 \mathrm{mmHg}^{(4)}$.

En caso de presentarse elevaciones de la misma, se habla de HIA y puede ser el reflejo de problemas abdominales subyacentes, representando un factor de riesgo independiente de mortalidad en el paciente crítico.

Varios estudios han confirmado que la presencia de HIA puede predecir la necesidad y la duración de la ventilación mecánica, así como la aparición de fallo de la función renal y la exigencia de terapia sustitutiva $^{(5)}$.

El valor exacto para definir la HIA no está claro: aunque se hable de valores entre 20 y $25 \mathrm{mmHg}$, con cifras próximas a 10 ó $15 \mathrm{mmHg}$ pueden darse alteraciones fisiopatológicas (el umbral está situado en la literatura entre 12 y $25 \mathrm{mmHg}$ ). Lo que está claro es que un valor elevado y aislado de PIA no vale para definir una HIA.

Son necesarias, por lo menos, tres mediciones (espaciadas 4 horas) con valores por encima de $12 \mathrm{mmHg}$.

El SCA, definido como el aumento sostenido de la PIA (>20 mmHg) acompañado de disfunción multiorgánica, hace referencia a un conjunto de síntomas y no a una enfermedad. Malbrain define el SCA como HIA gradual y mantenida, asociada a la disfunción de uno o más órganos, no presente previamente. El SCA es el resultado final de un aumento progresivo de la PIA. Al contrario de la HIA, el SCA no puede ser clasificado en estadios: existe o no existe. Se puede decir que la HIA es un proceso continuo con diferentes estadios, uno de ellos crítico (variable con cada paciente, cada situación y cada momento) y cuando éste se sobrepasa aparece en escena el SCA. Se habla de HIA cuando la PIA sobrepasa un determinado valor (generalmente situado entre 20 y $25 \mathrm{mmHg}$ ), y se habla de SCA cuando la HIA se acompaña de manifestaciones clínicas multisistémicas.

La incidencia real del SCA no está definida ya que depende del tipo de población de pacientes estudiados por los autores en los trabajos desarrollados al respecto y depende también del punto de corte establecido entre PIA normal e HIA. Se cifra la incidencia media de HIA en pacientes críticos en el 30-35\% va desde 19.8- 
$20 \%$ en pacientes con patología médica, hasta $30-39.4 \%$ en pacientes quirúrgicos de urgencia ${ }^{(6)}$.

\section{DISCUSIÓN}

La incidencia del SCA en pacientes críticos está cifrada en torno al 5\%. Es característica la presencia de altas presiones en la vía aérea durante la ventilación mecánica, hipoxia, oliguria y gasto cardíaco disminuido ${ }^{(7)}$.

El cuadro puede revertir si se aplica el tratamiento en el momento adecuado, siendo la descompresión abdominal quirúrgica la solución. Si el SCA no es sospechado, diagnosticado y tratado a tiempo, el resultado puede ser la muerte del paciente (tasa de mortalidad entre el 10.6 y el $71 \%$ en global, del $38 \%$ en pacientes traumatizados y $100 \%$ en pacientes con causa médica $)^{(8)}$.

\section{Etiopatogenia}

Los factores etiológicos más frecuentes son la administración masiva de fluidos $(77.5 \%)$, la infección intraabdominal (16.9\%), el hemoperitoneo $(14.1 \%)$, la cirugía abdominal (3.8\%), el pneumoperitoneo (1.4\%) y causas idiopáticas (7\%). Al menos dos factores se dan en el $52 \%$ de los pacientes.

Lo más importante es identificar los grupos de riesgo para tenerlos controlados de cerca. Se pueden incluir los politraumatizados graves a los que se haya practicado cirugía de control de daño y cierre primario de abdomen (con mortalidad del $85 \%$ si se instaura fallo multiorgánico), los pacientes que hayan sufrido una laparotomía por sangrado importante o isquemia intestinal, los pacientes que hayan sido sometidos a intervenciones quirúrgicas muy largas $\mathrm{y}$ complejas, pacientes con trauma abdominal y pélvico asociado (clínica en 4-5 horas e incidencia del 5.5\%).

En todos estos se desencadena la triada de hipotermia, acidosis y coagulopatía.

También se debe pensar en el SCA en grandes quemados (sobre todo aquellos con quemaduras importantes en toda la circunferencia del tórax), pacientes con hematomas retroperitoneales, lesiones ocupantes de espacio (tumores, obstrucciones intestinales), ascitis o pacientes con patología médica que hayan requerido grandes aportes de volumen para mantener su hemodinámica (entre 6 y 10 litros, dependiendo de los autores). En este tipo de pacientes suelen presentarse problemas a la hora del cierre de la pared abdominal por la presencia de edema de asas intestinales originado por los procesos de isquemia y reperfusión ${ }^{(9)}$.

\section{Clasificación}

El SCA se puede clasificar en:

Primario: si la causa es un traumatismo o una patología abdominal.

Secundario: si la causa es ajena al abdomen (amputación de miembro inferior, quemados, sepsis, lesiones penetrantes en tórax).

Terciario: si se desencadena a partir del tratamiento médico o quirúrgico de un SCA primario o secundario $^{(10)}$.

Otra manera de clasificarlo puede ser según la rapidez de instauración:

Hiperagudo: cuando la elevación de la PIA dura varios segundos o minutos tras un ejercicio físico o maniobras como la tos, estornudo o defecación.

Agudo: si se presenta a las pocas horas del postoperatorio inmediato tras un trauma abdominal o una hemorragia intraabdominal.

Subagudo: si tiene lugar tras varios días, sobre todo en pacientes médicos.

Crónico: cuando se desarrolla en
períodos de meses o años
$(2)$

\section{Fisiopatología}

Responde a una causa mecánica directa y dependerá de la velocidad de instauración de la misma. El SCA puede desarrollarse por un aumento de la PIA agudo o gradual; cuando ocurre esto último, los órganos afectados pueden ir adaptándose, pero cuando los cambios aparecen de forma aguda la respuesta suele ser insuficiente, con la consiguiente descompensación. En la mayoría de los casos es más importante la velocidad de instauración que el aumento absoluto de la PIA. También es importante el estado previo del paciente: la 
existencia de comorbilidades (insuficiencia renal crónica, hipovolemia, cardiomiopatía), hace que el umbral de PIA, que produce alteraciones fisiopatológicas, descienda y la tolerancia al aumento de la PIA (en un principio soportado) disminuya $^{(11)}$.

La HIA alta y sostenida conduce a un Síndrome de Disfunción Multiorgánica cuyas manifestaciones clínicas se deben a su repercusión en distintos parénquimas.

\section{Pulmonar}

Los cambios son puramente mecánicos. La HIA eleva los diafragmas, se comprimen los pulmones, aumenta la presión intratorácica y la presión pico máxima de la vía aérea, disminuye la distensibilidad pulmonar y se reduce la perfusión pulmonar, lo que produce hipoxia, hipercapnia y acidosis. La descompresión, habitualmente, mejora todos estos parámetros ${ }^{(12)}$.

\section{Cardiovascular}

A partir de una PIA entre 15-20 $\mathrm{mmHg}$, se observa una disminución del gasto cardíaco, al combinarse la disminución de la precarga cardíaca (compresión de la vena cava inferior), el aumento de la poscarga por la compresión de los órganos y vasos intraabdominales y la disminución de la distensibilidad ventricular. Y una PIA por encima de $30 \mathrm{mmHg}$, se añade una disminución de la contractilidad cardíaca al comprimirse las cavidades ${ }^{(12)}$.

\section{Renal}

Tiene un componente prerrenal por la disminución del gasto cardíaco y otro por compresión directa de los vasos renales, de modo que se produce una disminución del flujo arterial que condiciona la presión de perfusión renal y el gradiente de filtración glomerular. También se produce un aumento de las hormonas antidiuréticas y de las resistencias vasculares renales $^{(12)}$.

\section{Circulación esplácnica}

Las repercusiones gastrointestinales pueden ser muy tempranas, ya que con una PIA de 10 $\mathrm{mmHg}$ se reduce el flujo de sangre venosa portal y el de la arteria hepática, lo que supone alteraciones hepáticas en la síntesis de proteínas e inmunoglobulinas y factores de otros sistemas de defensa y con presiones de $20 \mathrm{mmHg}$ se alteran el flujo arterial mesentérico y el de la microcirculación en la zona de la mucosa intestinal. La isquemia mucosa resultante se traduce por una acidosis local, liberación de radicales libres de oxígeno, aumento de la permeabilidad capilar y translocación bacteriana que puede conducir a un choque séptico ${ }^{(12)}$.

\section{Mucosa gástrica}

Es una víctima temprana de la hipoperfusión y su isquemia es la primera repercusión clínicamente objetiva de la HIA. En la HIA hay una disminución de la perfusión mucosa y un aumento del metabolismo regional, con un desequilibrio entre el $\mathrm{CO}_{2}$ eliminado y el producido, cuyo resultado es una acumulación de $\mathrm{CO}_{2}$ en la mucosa gástrica, que puede detectarse tempranamente al realizar el seguimiento de la presión parcial de $\mathrm{CO}_{2}$. La acidosis intramucosa aporta información acerca de la perfusión mucosa y resulta una alarma temprana y una herramienta de gran utilidad en el diagnóstico y el tratamiento de la HIA y el SCA.

La determinación del $\mathrm{pH}$ intramucoso gástrico (pHimg) permite detectar la isquemia mesentérica del SCA, un pHimg-ácido es un factor de riesgo de mortalidad y hay una significación clínica entre PIA aumentada, > $20 \mathrm{mmHg}$ y el pHimg anormal $^{(12)}$.

\section{Deterioro de la pared abdominal}

La HIA reduce la perfusión sanguínea de los músculos de la pared, lo que crea un ambiente de isquemia y edema parietal, aumenta su rigidez y agrava la HIA, con riesgo a infección de la herida operatoria y evisceración ${ }^{(13)}$.

\section{Intracraneal}

Las consecuencias de una HIA en el sistema nervioso central son el aumento de la presión intracraneal y la disminución de la presión de perfusión cerebral $^{(24)}$.

La descompresión quirúrgica abdominal del SCA puede mejorar de forma espectacular la presión intracraneal y el estado neurológico del paciente en los traumatismos combinados de abdomen $\mathrm{y}$ tórax ${ }^{(12)}$. 


\section{Manifestaciones Clínicas}

Desde el punto de vista clínico, se caracteriza por distensión abdominal (PIA $>20 \mathrm{mmHg}$ ), hipoxia $\left(\mathrm{PaO}_{2} / \mathrm{FiO}_{2}<200\right)$, hipercapnia, presión en vías aéreas $>45 \mathrm{cmH}_{2} \mathrm{O}$, oliguria (diuresis $<0.5$ $\mathrm{ml} / \mathrm{kg} / \mathrm{h}$ o uremia), que suele ser la primera manifestación clínica y disminución del gasto cardíaco $\left(\mathrm{GC}<3 \mathrm{~L} \mathrm{~min} / \mathrm{m}^{2}\right.$ ), con un transporte de $\mathrm{O}_{2}\left(\mathrm{DO}_{2}\right)$ menor de $600 \mathrm{mlO} \mathrm{min}_{2} / \mathrm{m}^{2}$.

En ausencia de neumotórax y de obstrucción mecánica y en pacientes correctamente sedados y relajados, presiones pico elevadas en la ventilación mecánica son el factor predictivo único y más precoz del SCA.

El SCA está asociado con una mayor necesidad de ventilación mecánica, fallo renal y síndrome de distrés respiratorio, con el consiguiente aumento de estancia en las unidades de cuidados críticos. Está demostrado que no está asociado con mayor índice de abscesos intraabdominales, complicaciones biliares o infecciones de las heridas quirúrgicas.

Todas estas manifestaciones clínicas revierten con la descompresión quirúrgica del abdomen ${ }^{(20)}$. Si no se corrige con prontitud el aumento de la PIA, el resultado será el fallo multiorgánico y la muerte del paciente en el $100 \%$ de los casos $^{(14)}$.

\section{Medición de la Presión Intraabdominal}

Varios han sido los intentos de medir la PIA: vía indirecta, desde sondas vesicales, rectales o vaginales, manometría, presión en vena cava inferior (por vía femoral) o vía intragástrica (por tonometría), o, vía directa, de manera invasiva, mediante laparoscopia o mediante un catéter intraperitoneal (no apropiados para pacientes críticos por el riesgo aumentado de infección).

Todavía no existe un acuerdo para destacar a un método sobre los demás ${ }^{(15)}$.

El método de medición intravesical es el siguiente: la vejiga es vaciada y posteriormente rellenada con 50 (valor óptimo según Fusco27) o $100 \mathrm{ml}$ de suero fisiológico a temperatura ambiente (para establecer una columna de líquido que transmita la PIA). La sonda vesical se clampa distalmente al puerto de aspiración y se inserta una aguja de 16-gauge, conectada a su vez a un tubo con un sensor de presión, que será colocado a nivel de la sínfisis del pubis o de la línea medio-axilar y que enviará los datos a un monitor de presión. Para realizar una medición con este sistema se emplea mucho tiempo, lo cual supone un inconveniente, además de poner en peligro la esterilidad del sistema, con el consiguiente riesgo de infección y sepsis de origen urinario $^{(15)}$.

Después de la descripción de este método, varias han sido las modificaciones y hoy en día se acepta que el descrito por Cheathman (o su modificación) es el preferido: dos o tres llaves de tres pasos son interpuestas entre la sonda y el colector de orina. En una de las llaves está conectada una bolsa de suero fisiológico de 1000 $\mathrm{mL}$, en la otra, una jeringa de $60 \mathrm{~mL}$ y en la tercera, el transductor de presión. Las ventajas de este método son la rapidez, la seguridad, la facilidad para hacer mediciones repetidas, la mejor relación costo-beneficio y el menor riesgo de infección y de accidentes del personal. Se trata de la técnica más usada y es ideal para el "screening" y la monitorización durante cortos períodos de tiempo ${ }^{(15)}$.

Los métodos intragástricos (por sonda nasogástrica o por gastrostomía) tienen la ventaja de no depender de la posición del transductor de presión, ser baratos, no interfieren con la diuresis y no tienen riesgo de infección ni de pinchazos con el material. Pero también tienen inconvenientes, como la interferencia con la alimentación por la sonda nasogástrica o con el aire intragástrico (que debe ser aspirado) ${ }^{(15)}$.

La medición por sondas rectales o vaginales (intraútero) no tienen utilidad en el uso diario debido a que exigen más tiempo, mayor manipulación, son más difíciles, no pueden ser aplicadas en pacientes con sangrado digestivo bajo y diarrea (en el caso de las primeras) o con infección o sangrado ginecológico (en las segundas $)^{(15)}$.

La manometría utiliza la columna de orina del paciente como transductor de presión. Utiliza para ello el Foley-manómetro: un dispositivo de $50 \mathrm{ml}$ de capacidad es interpuesto entre la sonda urinaria y el colector de orina. El contenedor, que está lleno de orina, tiene que ser elevado para que los $50 \mathrm{ml}$ (volumen estándar) vuelvan a la vejiga del paciente. La columna de orina que 
queda en el dispositivo marcará la PIA del paciente $^{(28)}$. Este método permite mediciones repetidas, es rápido, tiene elevado costoefectividad y requiere poca manipulación ${ }^{(15)}$.

El principal inconveniente para la estimación de la PIA a través de la presión en vena cava inferior es la posibilidad de aparición de las complicaciones propias de la cateterización de un gran vaso (complicaciones mecánicas e infecciónsepsis $)^{(15)}$.

\section{Indicaciones para controlar la presión intraabdominal}

1. Postoperatorio de cirugía abdominal con cierre de la pared a tensión.

2. Traumas abdominales $\mathrm{y} / \mathrm{o}$ pélvicos graves abiertos o cerrados.

3. Cuadros clínicos con acumulación de líquidos intraabdominales.

4. Infecciones abdominales, peritonitis y pancreatitis.

5. Íleos paralíticos, mecánicos o subobstrucciones.

6. Pacientes con distensión abdominal $\mathrm{y}$ clínica del SCA.

7. Postoperatorio temprano de laparotomías con cierres temporales, para poder detectar un SCA silente.

8. En cualquier circunstancia en la que se administren masivamente fluidos, ya que se disminuye la perfusión intestinal y aumenta la incidencia de HIA, SCA y SDMO

9. Pacientes de UCI con ventilación mecánica y SDMO.

10. Neumoperitoneo y hemorragias retroperitoneales $^{(2)}$.

\section{Tratamiento}

La actitud terapéutica recomendada por Meldrum y Moore, según el grado de HIA, es la siguiente :

- $10-15 \mathrm{mmHg}$ de PIA (SCA grado I) = medidas de mantenimiento (no suele tener consecuencias);

- $16-25 \mathrm{mmHg}$ de PIA (SCA grado II) = aumento del aporte de volumen para sustento hemodinámico

- $\quad$ 26-35 mmHg de PIA (SCA grado III) = descompresión quirúrgica (sin llegar a ser urgente);
- $\quad>35 \mathrm{mmHg}$ (SCA grado IV) = descompresión quirúrgica y reexploración inmediatas.

La primera aproximación a este síndrome se lleva a cabo desde el punto de vista médico, sin demorar en ningún caso el tratamiento quirúrgico.

\section{Tratamiento médico}

Las medidas terapéuticas médicas en el SCA son limitadas, sus objetivos son:

- Mantener una adecuada PPA > de 50 $\mathrm{mmHg}$.

- Mejorar la ventilación y el aporte de oxígeno.

- Tratar los defectos de la coagulación.

- Mantener un llenado vascular adecuado, con soluciones cristaloides, manitol y bicarbonato en caso necesario, antes de descomprimir el abdomen, para prevenir la hipotensión reactiva a la disminución de la PIA.

- Otras medidas, si se requieren, como la paracentesis, la aspiración nasogástrica y/o rectal, los enemas rectales, el uso de procinéticos, la hemofiltración continua, la furosemida y albúmina humana al $20 \%$ y la ventilación mecánica con sedación y relajación.

Como alternativas terapéuticas novedosas, están la disminución no invasiva de la PIA, en la que se aplica una presión negativa extraabdominal $^{(25)}$ y el drenaje percutáneo de colecciones en la cavidad abdominal y/o retroperitoneo $^{(16)}$.

\section{Tratamiento quirúrgico}

La descompresión quirúrgica del abdomen (laparostomía) es el único tratamiento definitivo del SCA. Tiene una efectividad del $93 \%$ en la regresión del fallo multiorgánico y ofrece una supervivencia del 59\%.

Desde el punto de vista quirúrgico existen dos actitudes: aplicar el tratamiento preventivo, con aplicación de la "Bolsa de Bogotá" o cualquier otra técnica en una primera fase; o realizar un cierre normal, esperar y ver, realizando una 
segunda laparotomía en caso de presentarse el SCA.

Lo controversial del tratamiento quirúrgico del SCA se sitúa en cuándo se debe llevar a cabo? Los niveles de PIA a los que se desarrolla un SCA dependen de cada paciente. Unos autores defienden este tratamiento con cifras de PIA superiores $22 \mathrm{mmHg}$; sin embargo otros proponen cifras mucho más bajas (próximas a 10 $\mathrm{mmHg}$ ), en las que ya se pueden encontrar signos de disfunción orgánica. Burch y Biffl han sugerido el tratamiento quirúrgico indiscutible para aquellos casos de HIA grado III y IV ${ }^{(17)}$.

Será necesario adecuar el estado del paciente antes de someterlo al tratamiento quirúrgico. Es importante corregir la hipotermia, la coagulación, la acidosis metabólica y la volemia.

Cuando se haya descomprimido el abdomen es importante no cerrarlo a tensión, lo que ayudará también a mantener la integridad de los músculos y la fascia para un cierre a posteriori. El cierre a tensión desemboca en isquemia de la fascia, necrosis, infección y dehiscencia de la pared abdominal, acompañada de una mortalidad próxima al $90 \%$ de los pacientes. La probabilidad de aparición del SCA es 11 veces mayor si se realiza el cierre fascial primario que si se deja descomprimido $^{(18)}$.

Debe evitarse dejar el abdomen abierto por el riesgo de complicaciones que conlleva, tales como la pérdida excesiva de fluidos (próxima a la pérdida de un paciente con quemaduras de tercer grado en el $50 \%$ de su cuerpo), la formación de fístulas, el uso prolongado de relajantes musculares para evitar la evisceración y permitir la ventilación mecánica, o el daño de la piel del abdomen por los fluidos abdominales (con problemas en la reconstrucción final de la pared).

Para evitar estas complicaciones y permitir el cierre abdominal libre de tensión, existen varias posibilidades:

Bolsa de Bogotá: bolsa de irrigación urológica cosida a las paredes abdominales, que descansa sobre el contenido visceral y permite ver el estado de las mismas, descrita por primera vez por Londoni en Bogotá (Colombia); mallas reabsorbibles: de ácido poliglicólico. Deben colocarse con el epiplón separándolas de las vísceras.

Mallas no reabsorbibles: Gore-Tex (principalmente), Marlex y Silastic. Presentan bajo riesgo de fístula intestinal y hernia (menor que las reabsorbibles).

“Vacuum pack": cierre al vacío, con material de polietileno, con aspiración a través de drenajes; cierres con cremallera, que permiten la reexploración intraabdominal ${ }^{(19)}$.

También están descritas técnicas descompresivas mínimamente invasivas, como la laparoscopia o los procedimientos percutáneos apoyados por ultrasonidos, si se estima que los pacientes no toleran la cirugía abierta, pero no son de uso extendido $^{(20)}$.

El decidir llevar a cabo la descompresión abdominal quirúrgica conlleva el traslado del paciente al quirófano. Usualmente el paciente no está en óptimas condiciones, por lo que en ocasiones tendrá que valorarse la realización de la técnica en la misma unidad de críticos. Se debe disponer de todos los medios necesarios para realizar la reanimación del paciente en caso de presentarse complicaciones ${ }^{(21)}$.

El cierre definitivo de la pared abdominal se hará cuando se haya optimizado el estado del paciente, cuando se pueda realizar la aproximación de las fascias y cuando el riesgo de resangrado y nueva laparotomía sea mínimo ${ }^{(21)}$.

\section{CONCLUSIONES Y RECOMENDACIONES}

Resulta más importante la rapidez de instauración del cuadro (con limitación del tiempo de acomodación a la situación) que las cifras absolutas de la PIA.

Conocer y pensar en la HIA permite su diagnóstico temprano y un tratamiento adecuado, ya que un SCA establecido sin un tratamiento correcto es una complicación mortal, en la mayoría de los casos, por SDMO.

Por lo general, el tratamiento quirúrgico del paciente con SCA desemboca en la mejoría clínica del paciente de manera inmediata (si se realiza a tiempo). 


\section{REFERENCIAS BIBLIOGRÁFICAS}

1. Kron IL Harman PK Nolan SP. The measurement of intra-abdominal pressure as a criterion for abdominal reexploration. Ann Surg. 1984;199:28-30.

2. Abdominal Compartment Syndrome. World Congress. Abstract Book. NoosaQueensland-Australia; 2004.

3. Bailey J Shapiro MJ. Abdominal compartment syndrome. Crit Care 2000;4:(4):23-29.

4. Moore AFK Hargest R Martin M Delicata RJ. Intra-abdominal hypertension and the abdominal compartment syndrome. Br J Surg. 2004;91(9):11021110.

5. Malbrain MLNG. Abdominal pressure in the critically ill: measurement and clinical relevance. Intensive Care Med. 1999;25(12):1453-1458.

6. Sugrue M. Abdominal compartment syndrome. Curr Opin Crit Care. 2005;11(4):333-338.

7. Meldrum DR Moore FA Moore EE Franciose RJ Sauaia A Burch JM. Prospective characterization and selective management of the abdominal compartment syndrome. Am J Surg. 1997;174(6):667-673.

8. McNelis J Soffer S Marini CP et al. Abdominal compartment syndrome in the surgical intensive care unit. Am Surg. 2002;68(1):18-23.

9. Ertel W Oberholzer A Platz A Stocker $\mathrm{R}$ Trentz O. Incidence and clinical pattern of the abdominal compartment syndrome after "damage-control" laparotomy in 311 patients with severe abdominal and/or pelvic trauma. Crit Care Med. 2000;28(6):1747-1753.

10. Maxwell RA Fabian TC Croce MA Davis KA. Secondary abdominal compartment syndrome: an underappreciated manifestation of severe hemorrhagic shock. J Trauma. 1999;47(6):995-999.

11. Burch JM Ortiz VB Richardson RJ Martin RR Mattox KL Jordan GL Jr. Abbreviated laparotomy and planned reoperation for critically injured patients. Ann Surg. 1992;215:476-483.

12. Morken J West MA. Abdominal compartment syndrome in the intensive care unit. Curr Opin Crit Care. 2001;7:268274.
13. Saggi BH Sugerman HJ Ivatury RR Bloomfield GL. Abdominal compartment syndrome. J Trauma. 1998;45:597609.

14. Raeburn CD Moore EE Biffl WL et al. The abdominal compartment syndrome is a morbid complication of post-injury damage control surgery. Am J Surg. 2001;182(6):542-546.

15. Malbrain ML. Different techniques to measure intra-abdominal pressure (IAP): time for a critical re-appraisal. Intensive Care Med. 2004;30(3):357371.

16. Latenser BA Kowal-Vern A Kimball D Chakrin A Dujovny N. A pilot study comparing percutaneous decompression with descompressive laparotomy for acute abdominal compartment syndrome in thermal injury. J Burn Care Rehábil. 2002;23:190-195.

17. Biffl WL Moore EE Burch JM Offner PJ Franciose RJ Jonson JL. Secondary abdominal compartment syndrome is a highly lethal event. Am J Surg. 2001;182(6):645-648.

18. Offner PJ De Souza AL Moore EE et al. Avoidance of abdominal compartment syndrome in damagecontrol laparotomy after trauma. Arch Surg. 2001;136(6): 676-681.

19. Mayberry JC Mullins RJ Crass RA Trunkey DD. Prevention of abdominal compartment syndrome by absorbable mesh prosthesis closure. Arch Surg. 1997;132(9):957-962.

20. Reckard JM Chung MH Varma MK Zagorski SM. Management of intraabdominal hypertension by percutaneous catheter drainage. J Vasc Interv Radiol. 2005;16(7):1019-1021.

21. Nathens AB Brenneman FD Boulanger $\mathrm{BR}$. The abdominal compartment syndrome. Can J Surg. 1997;40(4):254-258

\section{DECLARACIÓN DE CONFLICTO DE IN- TERESES}

Los autores declaran que no existen conflictos de intereses. 